\title{
Cardiopatia Congénita em Crianças com Síndrome de Down: $O$ que Mudou nas Últimas Três Décadas?
}

\section{Congenital Heart Disease in Children with Down Syndrome: What Has Changed in the Last Three Decades?}

\author{
Filipa Mestre A. DIAS $\otimes^{1}$, Susana CORDEIRO², Isabel MENEZES ${ }^{2}$, Graça NOGUEIRA², Ana TEIXEIRA², \\ Marta MARQUES ${ }^{3}$, Miguel ABECASIS ${ }^{3}$, Rui ANJOS ${ }^{2}$ \\ Acta Med Port 2016 Oct;29(10):613-620 - http://dx.doi.org/10.20344/amp.7318
}

\section{RESUMO}

Introdução: A prevalência da síndrome de Down tem aumentado nos últimos 30 anos; $55 \%$ destas crianças apresentam cardiopatia congénita.

Material e Métodos: Estudo retrospetivo longitudinal de coorte; dados clínicos obtidos em bases de dados de 1982 a 2013 com o diagnóstico de síndrome de Down ou trissomia 21 num hospital de referência em cardiologia pediátrica e cirurgia cardíaca.

Objetivo: Avaliar a evolução, nas últimas três décadas, dos cuidados cardiológicos prestados às crianças com síndrome de Down e cardiopatia congénita.

Resultados: Estudámos 102 doentes com síndrome de Down e cardiopatia congénita submetidos a terapêutica invasiva: cirurgia cardíaca corretiva, paliativa e cateterismo terapêutico. Em doentes referenciados no primeiro ano de vida, a referenciação foi cada vez mais precoce. O diagnóstico mais frequente foi o defeito completo do septo aurículo-ventricular (41\%). Verificou-se uma tendência para cirurgia corretiva cada vez mais precoce em doentes abaixo dos 12 meses $(p<0,001)$. A partir de 2000 , a grande maioria dos doentes foi operada antes dos seis meses de idade. As principais complicações cardíacas foram alterações de ritmo e baixo débito e as principais não cardíacas foram pulmonares e infeciosas. A taxa de mortalidade a 30 dias foi de 3/102 casos (2,9\%). Dos doentes em follow-up, 89\% estão em classe funcional I da NYHA.

Discussão e Conclusão: A correção cirúrgica mais precoce verificada nos últimos 15 anos vai ao encontro do proposto na literatura. A taxa de mortalidade a 30 dias verificada é sobreponível aos resultados internacionais. Os doentes com síndrome de Down submetidos a cirurgia corretiva de cardiopatia congénita apresentam uma excelente capacidade funcional a longo prazo.

Palavras-chave: Cardiopatias Congénitas; Procedimentos Cirúrgicos Cardíacos; Resultado do Tratamento; Síndrome de Down.

\section{ABSTRACT}

Introduction: The prevalence of Down syndrome has increased in the last 30 years; $55 \%$ of these children have congenital heart disease.

Material and Methods: A retrospective longitudinal cohort study; clinical data from 1982 to 2013 databases with the diagnosis of Down syndrome or trisomy 21 in a reference hospital in pediatric cardiology and cardiac surgery.

Objective: to assess the progress in the last three decades of cardiological care given to children with Down syndrome and congenital heart disease.

Results: We studied 102 patients with Down syndrome and congenital heart disease subjected to invasive therapy: corrective or palliative cardiac surgery and therapeutic catheterization. The referral age was progressively earlier in patients referred in the first year of life. The most frequent diagnosis was complete atrioventricular sptal defect $(41 \%)$. There was a trend towards increasingly early corrective surgery in patients under 12 months $(p<0.001)$. Since 2000 , the large majority of patients were operated before reaching six months of age. The main cardiac complications were rhythm dysfunction and low output. More frequent noncardiac complications were pulmonary and infectious. The 30 -day mortality rate was 3/102 cases (2.9\%). Of patients in follow-up, $89 \%$ are in NYHA class I. Discussion and Conclusion: The early surgical correction seen over the past 15 years follows the approach suggested in the literature. The observed 30-day mortality rate is overlapping international results. Patients with Down syndrome subjected to corrective surgery of congenital heart disease have an excellent long-term functional capacity.

Keywords: Cardiac Surgical Procedures; Down Syndrome; Heart Defects, Congenital; Treatment Outcome.

\section{INTRODUÇÃO}

A síndrome de Down (SD), caracterizada geneticamente pela trissomia de todo ou de uma porção crítica do cromossoma 21, é a anomalia cromossómica mais comum em nados-vivos. ${ }^{1}$ A trissomia 21 associa-se a alterações fenotípicas e a manifestações sistémicas características e a sua prevalência tem aumentado significativamente: $25 \%$ nos últimos 30 anos, $^{2}$ em relação com o aumento da idade materna.

Quarenta a cinquenta por cento das crianças com SD apresentam cardiopatia congénita, sendo as mais frequentes o defeito completo ou incompleto do septo aurículo-ventricular (DSAV), defeitos do septo auricular ou ventricular e a tetralogia de Fallot (ToF). ${ }^{3}$ Por outro lado, o desenvolvimento de hipertensão pulmonar é também muito mais frequente no SD, o que se deve a um menor número de unidades alveolares, à menor espessura da túnica média das arteríolas e à disfunção endotelial, mais frequente nestes doentes. ${ }^{4}$ Apesar destes factos, a mortalidade hospitalar

1. Serviço de Pediatria. Unidade de Faro. Centro Hospitalar do Algarve. Faro. Portugal.

2. Serviço de Cardiologia Pediátrica. Hospital de Santa Cruz. Centro Hospitalar de Lisboa Ocidental. Lisboa. Portugal.

3. Serviço de Cirurgia Cardiotorácica. Hospital de Santa Cruz. Centro Hospitalar de Lisboa Ocidental. Lisboa. Portugal.

$\square$ Autor correspondente: Filipa Mestre A. Dias. ana.filipa.mestre@gmail.com

Recebido: 16 de setembro de 2015 - Aceite: 12 de setembro de 2016 | Copyright $\odot$ Ordem dos Médicos 2016 
associada a cirurgia cardíaca corretiva é significativamente menor para os doentes com SD quando comparados com doentes sem SD. ${ }^{5}$

Os doentes com SD apresentam complicações a nível de outros órgãos ou sistemas com prevalência superior à da população geral, das quais se destacam obesidade, hipotiroidismo, maior probabilidade de desenvolvimento de neoplasias hematológicas (com um perfil genético e de resposta à quimioterapia particulares), maior risco de infeção (em parte devido a défices específicos de imunoglobulinas), assim como maior risco de autoimunidade. ${ }^{4}$

A população com SD constitui assim cada vez mais um grupo particular dentro de toda a medicina, uma vez que a sua prevalência e sobrevida têm aumentado significativamente ao longo dos anos. A cirurgia de cardiopatias complexas em crianças com SD só começou a ser realizada a partir dos anos 70 do século passado. Antes da década de 80 , a aceitação social das crianças com SD levantava muitas questões, sendo as mesmas frequentemente institucionalizadas. A partir dessa data, coincidindo com o advento da ecocardiografia, os cuidados prestados a estas crianças na área da cardiologia e cirurgia cardíaca melhoraram significativamente. ${ }^{6}$ As crianças com SD e cardiopatia congénita passaram a ser operadas cada vez mais cedo, com resultados cirúrgicos e mortalidade sobreponíveis aos das crianças sem SD.7,8

\section{MATERIAL E MÉTODOS}

Estudo retrospetivo longitudinal de coorte, realizado a partir de dados clínicos dos processos de doentes selecionados em bases de dados de cirurgias e de cateterismos cardíacos desde 1982 até 2013 com o diagnóstico de síndrome de Down ou trissomia 21 num hospital de referência em cardiologia pediátrica e cirurgia cardíaca. Destes, foram excluídos os doentes com dados omissos, com SD sem cardiopatia ou com cardiopatia minor, sem necessidade de intervenção. Dos restantes, apenas foram selecionados para este estudo os doentes com cardiopatia congénita submetidos a terapêutica por cateterismo cardíaco ou cirurgia.

O principal objetivo deste estudo foi avaliar se ao longo das últimas três décadas existiram diferenças significativas em termos de cuidados cardiológicos prestados às crianças com SD e cardiopatia congénita.

Para a recolha dos dados foram consideradas as variáveis referentes ao motivo de referenciação, diagnósticos, complicações e follow-up.

Para avaliação da presença de hipertensão arterial pulmonar (HAP) foram considerados os dados ecográficos sugestivos de HAP para as patologias que cursam com shunt esquerdo-direito, na ausência de estenose pulmonar, e os resultados do estudo hemodinâmico nos casos submetidos a cateterismo diagnóstico.

Relativamente à cirurgia corretiva foram consideradas as seguintes variáveis: 1. Idade; 2. Peso (kg); 3. Complicações cardíacas; 4. Complicações extra-cardíacas. Nas complicações respiratórias foram incluídas: extubação difí- cil/ventilação prolongada, atelectasia, parésia diafragmática, quilotórax, hemotórax, pneumotórax. Nas complicações infeciosas foram consideradas: sépsis, bacteriemia (hemocultura positiva), infeções com ponto de partida no cateter, pneumonia. Nas complicações da ferida operatória foram consideradas infeção da ferida (sem clínica de bacteriemia/ sépsis) e deiscência da sutura. Nas complicações metabólicas foram incluídas: hiperglicemia com necessidade de insulinoterapia, hipoglicemia, hiperbilirrubinemia. Nas complicações hematológicas foram incluídas: trombocitopenia, anemia com necessidade de suporte transfusional. Nas complicações renais foram incluídas: oligúria, falência renal com necessidade de substituição da função renal com diálise peritoneal ou hemodiafiltração. Nas complicações neurológicas foram consideradas convulsões, défices neurológicos de novo ou sinais de hemorragia/isquemia em exame imagiológico do sistema nervoso central. Foi considerada disfunção multiorgânica quando três ou mais órgãos/sistemas estiveram envolvidos. A presença de HAP precoce (primeiro mês pós-operatório), e a médio prazo (após o primeiro mês e até ao $12^{\circ}$ mês pós-operatório) foi avaliada por estimativa da pressão na artéria pulmonar através de ecocardiograma. Foi considerada reoperação precoce se foi realizada no primeiro mês de pós-operatório inicial e reoperação tardia se realizada após o primeiro mês. Pela consulta dos processos clínicos recolheu-se a classe funcional (NYHA) à data da última consulta. Foi ainda registada a ocorrência de falecimento e a data do mesmo.

A análise estatística foi realizada utilizando o software $\mathrm{R}^{\circledR}$ versão 3.1.2, SPSS versão 21 e Excel. A relação entre duas variáveis binárias foi estudada através do teste do qui-quadrado da independência ou teste de Fisher se amostras com contagem inferior a 20 , enquanto a relação entre uma variável binária e uma variável contínua ou de contagem foi avaliada através de modelos de regressão logística e testes da razão de verosimilhanças.

Considerou-se como estatisticamente significativos os resultados com valor de $p<0,05$.

\section{Limitações}

O presente estudo decorreu num centro de referência em cardiologia pediátrica e cirurgia cardíaca, pelo que as suas conclusões podem de certo modo estar condicionadas pela natureza da instituição.

Pela sua natureza retrospetiva, este trabalho apresenta limitações, nomeadamente no que respeita ao registo de todas as complicações ocorridas e da classificação funcional dos doentes durante o seguimento. A consulta de processos clínicos em papel, alguns com mais de 20 anos, dificultou a colheita de dados, que procurou no entanto ser o mais rigorosa possível.

\section{RESULTADOS \\ Caracterização da população}

Estudámos 102 doentes com SD submetidos a intervenção terapêutica invasiva (cirurgia paliativa, corretiva e cateterismo terapêutico) no contexto de cardiopatia congénita. 
Foi efetuada uma análise comparativa dos dados referentes aos doentes que foram submetidos a cirurgia cardíaca corretiva (85 doentes). Dos restantes doentes, 15 foram submetidos a cateterismo terapêutico e dois foram submetidos apenas a realização de cirurgia paliativa, sem realização de cirurgia corretiva. Não foram incluídos três doentes referenciados tardiamente que foram considerados inoperáveis por hipertensão pulmonar.

Na totalidade da amostra estudada, 66 doentes $(64,7 \%)$ eram do sexo feminino, tendo sido referenciados entre fevereiro de 1982 e junho de 2013.

As cirurgias corretivas ocorreram entre fevereiro de 1986 e outubro de 2013.

Cinquenta e nove doentes foram referenciados por sinais clínicos: 36 (35\%) por sopro, 20 (19,6\%) por insuficiência cardíaca, três $(2,9 \%)$ por cianose; $12(11,8 \%)$ doentes foram referenciados para rastreio de doença cardíaca, quatro $(3,9 \%)$ por diagnóstico pré-natal de cardiopatia, 17 $(16,6 \%)$ por ecocardiograma pós-natal revelando cardiopatia, sete $(6,8 \%)$ referenciados pela consulta de Cardiologia Pediátrica de outro serviço para cirurgia cardíaca. Não foram encontrados registos relativos ao motivo de referenciação dos restantes três doentes (Fig. 1).

Verificou-se um aumento estatisticamente significativo da referenciação por diagnóstico pré-natal nos últimos anos (teste $\mathrm{F}$ da ANOVA com $p=0,014$ ). Os quatro casos em questão foram referenciados após 2011, todos eles com o diagnóstico ecográfico pré-natal de defeito completo do septo aurículo-ventricular (DCSAV), um dos quais associado a ToF.

A média da idade de referenciação situou-se nos 18,6 meses, mediana de 48 dias [IQR (13 - 221) dias], com mínimo no primeiro dia de vida e máximo aos 36,8 anos.
Para doentes referenciados antes dos 12 meses de vida (69 doentes), verificou-se uma tendência para uma referenciação cada vez mais precoce a cada ano que passou (teste $\mathrm{F}$ da ANOVA com $p=0,030$ ). A distribuição da idade de referenciação para doentes referenciados antes dos 12 meses foi significativamente diferente ( $\mathrm{F}$ da ANOVA, $p=$ $0,015)$ para doentes referenciados antes e depois de janeiro de 1994. Assim, antes de 1994, a idade de referenciação apresentava uma mediana de 153 dias e após 1994 (inclusive) a mediana passou a situar-se nos 31 dias de vida.

Apenas 43 doentes (42\%) apresentavam cardiopatia congénita isolada (um único diagnóstico), sendo que os restantes associavam dois ou mais diagnósticos.

A distribuição por diagnósticos principais pode ver-se na Tabela 1.

\section{Doentes submetidos a cateterismo terapêutico}

Quinze doentes foram tratados por cateterismo terapêutico, sem intervenção cirúrgica. Estes doentes apresentavam como diagnóstico principal: persistência do canal arterial $(P C A)-n=7$, comunicação interauricular do tipo ostium secundum (CIA-OS) - $\mathrm{n}=6$, comunicação interventricular $(\mathrm{CIV})-\mathrm{n}=2$.

A idade de intervenção foi em média de 32 meses, mediana de 16 meses [IQR (11 - 65) meses].

Não se verificou variação significativa do número de doentes tratados por cateterismo terapêutico ao longo dos anos (teste da razão de verosimilhanças, $p=0,25$ ).

\section{Doentes submetidos a cirurgia}

Oitenta e sete doentes foram submetidos a cirurgia cardíaca. Em 85 casos tratou-se de cirurgia corretiva e em nove casos de cirurgia paliativa. Dois doentes foram Sem dados

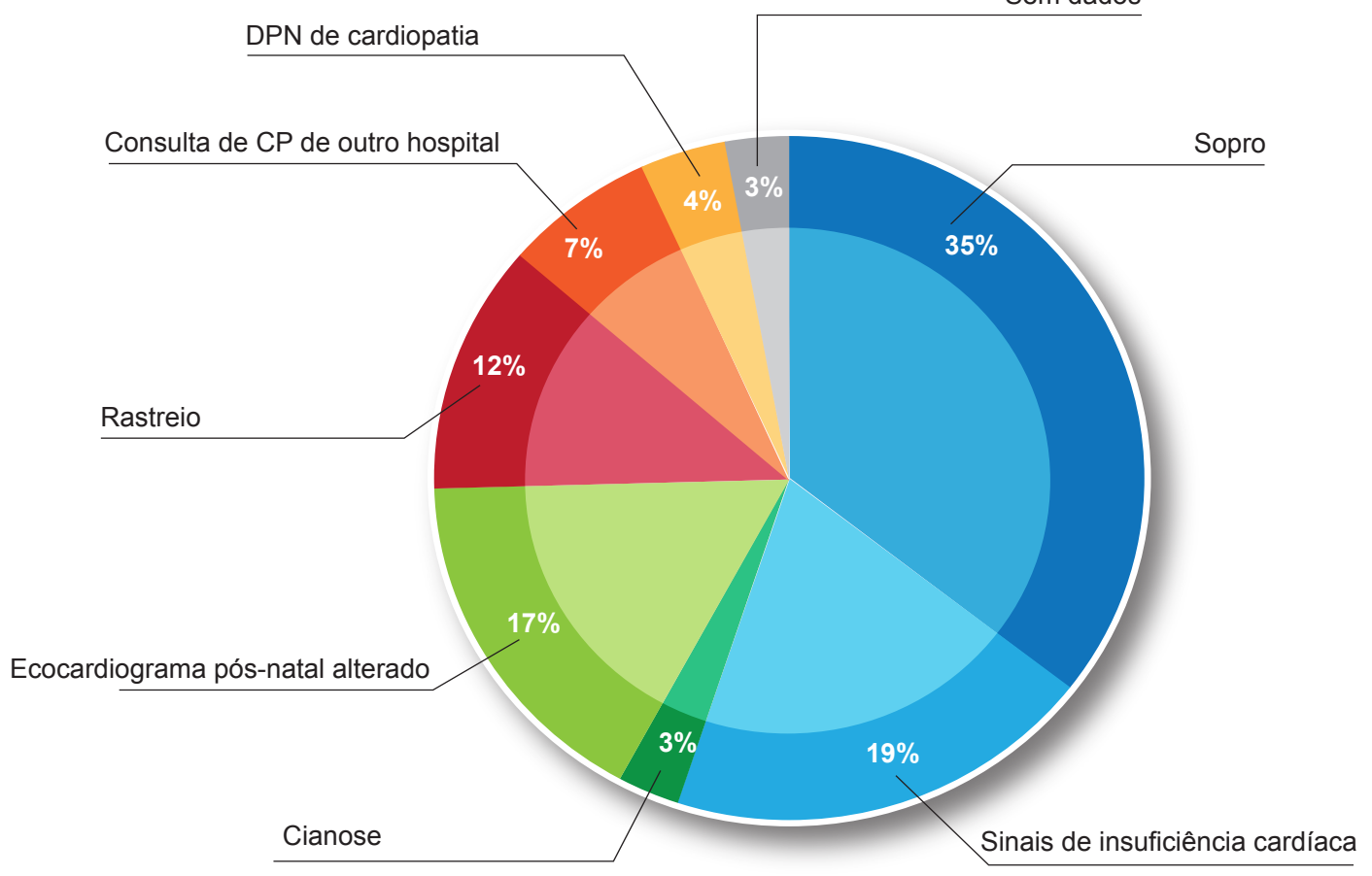

Figura 1 - Distribuição dos motivos de referenciação para o hospital de referência em cardiologia pediátrica 
Tabela 1 - Cardiopatias congénitas por diagnóstico principal

\begin{tabular}{lcc}
\hline Cardiopatia & $\mathbf{n}$ & $\mathbf{( \% )}$ \\
\hline DCSAV & 42 & $(41,2)$ \\
DCSAV simples & 36 & \\
DCSAV + TOF & 4 & \\
DCSAV + CoAo & 2 & \\
DISAV & 5 & $(4,9)$ \\
CIV & 32 & $(31,4)$ \\
CIA-OS & 11 & $(10,8)$ \\
TOF & 5 & $(4,9)$ \\
PCA & 7 & $(6,9)$ \\
Total & 102 & \\
\hline
\end{tabular}

DCSAV: Defeito completo do septo aurículo-ventricular; DISAV: Defeito incompleto do septo aurículo-ventricular; TOF: Tetralogia de Fallot; CIV: Comunicação interventricular; CIA-OS: Comunicação interauricular do tipo ostium secundum; CoAo: Coartação da aorta; PCA: Persistência do canal arterial

submetidos a cirurgia paliativa sem correção cirúrgica posterior.

\section{Pré-cirurgia}

Dos 87 doentes submetidos a cirurgia, $42(48 \%)$ foram submetidos a cateterismo diagnóstico prévio. Cinquenta destes doentes $(57 \%)$ apresentavam HAP pré-cirúrgica, entre os 34 em que foi confirmada por cateterismo.

Verificou-se uma relação estatisticamente significativa entre o ano da realização da cirurgia corretiva e a realização de cateterismo de diagnóstico prévio (teste da razão de verosimilhanças com $p<0,01$ ). O modelo de regressão logística simples revelou 1,49 vezes menor probabilidade de realização de cateterismo diagnóstico a cada ano que passou (IC 95\%).

\section{Cirurgia paliativa}

Os principais motivos para realização de cirurgia paliativa foram: peso considerado baixo para correção cirúrgica ou a existência de comorbilidades significativas, entre elas HAP (para avaliação da resposta das pressões da artéria pulmonar ao banding, com vista à cirurgia corretiva posterior).

Sete doentes foram submetidos a cirurgia paliativa antes da cirurgia corretiva, dos quais quatro corresponderam a anastomoses de Blalock-Taussig e três a banding da artéria pulmonar, um destes últimos com correção simultânea de interrupção do arco aórtico. Estes doentes foram submetidos a cirurgia corretiva em média sete meses após cirurgia paliativa, com mediana de 11 meses $\operatorname{IQR}(6,15)$ meses].

Dois doentes foram submetidos apenas a cirurgia paliativa, sem realização de cirurgia corretiva. Ambos apresentavam DCSAV, tendo realizado banding da artéria pulmonar com 50 e 59 dias de vida. Um dos casos faleceu 32 dias após a cirurgia por causa não cardíaca, após transferência para o hospital da área de residência. $\mathrm{O}$ outro caso foi per- dido para follow-up.

\section{Cirurgia corretiva}

A idade média em que os doentes foram submetidos a cirurgia corretiva foi de 37 meses, com mediana de nove meses [IQR $(6,25,5)$ meses]. Nos doentes com menos de 12 meses de vida $(n=49)$, verificou-se uma tendência para uma idade cada vez mais precoce à data de cirurgia (teste $F$ da ANOVA com $p<0,001)$. Nos últimos 13 anos de estudo (2000 - 2013), foram submetidos a cirurgia corretiva 24 doentes com menos de 12 meses de idade, com os diagnósticos principais de DCSAV ou CIV. Estes foram todos operados antes dos oito meses de idade, em média com 5,1 meses, mediana de seis meses [IQR $(4,6,75)$ meses]. Desta forma conclui-se que a maioria dos doentes foi operada antes dos 6 meses de idade (Fig. 2).

A média de peso dos doentes à data de cirurgia corretiva era de $10,8 \mathrm{~kg}$, mediana $6,4 \mathrm{~kg}$ [IQR $(5,1-10,1) \mathrm{kg}$, mínimo de $3 \mathrm{~kg}$. Nos doentes com menos de $8 \mathrm{~kg}(\mathrm{n}=51)$, verificou-se uma tendência para um peso cada vez mais baixo à data de cirurgia (teste $\mathrm{F}$ da ANOVA $\operatorname{com} p=0,027$ ).

\section{Pós-operatório de cirurgia corretiva}

Foi estudada a relação entre a HAP pré-cirúrgica, no pós-operatório imediato/precoce transitória (primeiro mês), a médio prazo (um a 12 meses após cirurgia) e tardia (após 12 meses).

Dos 85 doentes submetidos a cirurgia corretiva, 48 doentes apresentavam HAP pré-cirúrgica e 24 doentes apresentaram HAP precoce transitória no pós-operatório imediato. Quatro doentes apresentaram HAP a médio prazo, dois pela presença de CIVs residuais e dois por insuficiência da válvula aurículo-ventricular (AV) esquerda, que motivaram reoperação tardia. Não houve registo de qualquer doente operado que mantivesse HAP tardia persistente por doença vascular pulmonar.

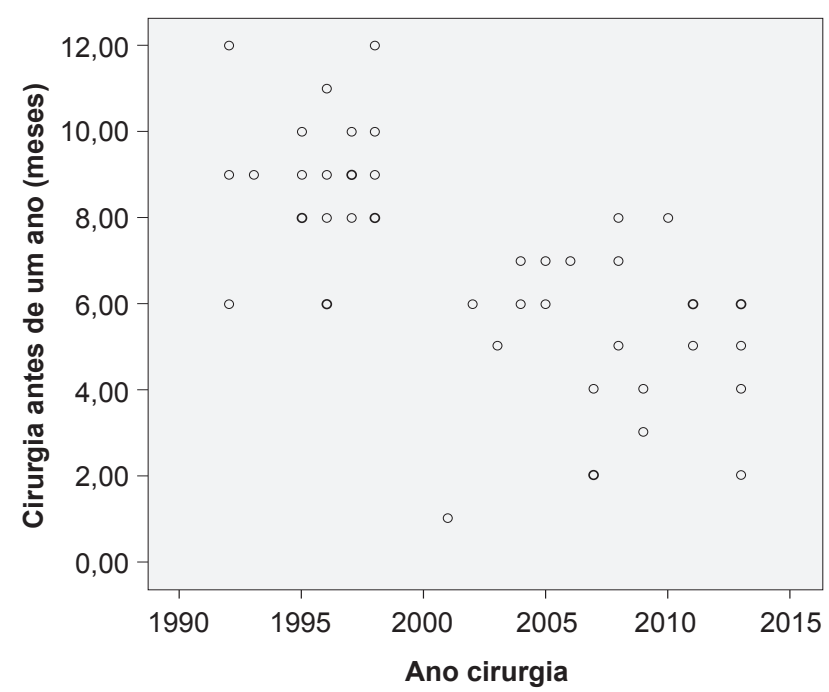

Figura 2 - Gráfico representativo da distribuição das idades (para doentes com idade < 12 meses) à data de cirurgia corretiva ao longo dos anos de estudo 
Observou-se uma relação significativa entre a HAP pré cirúrgica e no pós-operatório imediato $\left(\chi^{2}, p=0,03\right)$. Por outro lado, a existência de HAP pré-cirúrgica não apresentou uma relação significativa com a existência de HAP a médio prazo ou HAP tardia persistente.

Verificou-se uma maior prevalência de HAP precoce transitória com o aumento da idade à data de cirurgia para os doentes operados antes dos 12 meses de vida, apesar de não significativa (teste $F$ da ANOVA com $p=0,074$ ) e ainda uma tendência para diminuição da ocorrência de HAP precoce transitória com o ano de cirurgia (razão de verosimilhanças, $p=0,042$ e $\beta$ negativo).

Relativamente a complicações pós-operatórias nos doentes submetidos a cirurgia corretiva, 15 apresentaram complicações cardíacas e 44 complicações não cardíacas. Dez doentes associaram complicações cardíacas e não cardíacas. Verificou-se que a ocorrência de complicações cardíacas não esteve relacionada com a ocorrência de complicações não cardíacas $\left(\chi^{2}\right.$, valor $\left.p=0,81\right)$.

As principais complicações cardíacas verificadas foram alterações de ritmo e baixo débito. Cerca de $26 \%$ dos doentes apresentaram complicações pulmonares $(n=22)$ e $18 \%$ apresentou complicações infeciosas $(n=15)$. A distribuição dos tipos de complicações pode ver-se na Tabela 2.

Verificou-se ausência de relação estatisticamente significativa entre a presença de complicações cardíacas e o tipo de cardiopatia congénita (razão de verosimilhanças, $p$ $=0,7)$.

Observou-se uma relação estatisticamente significativa entre o tipo de cardiopatia congénita e as complicações não cardíacas $\left(\chi^{2}, p=0,025\right)$. O modelo de regressão entre complicações não cardíacas e cada tipo de cardiopatia revelou uma probabilidade 2,66 vezes menor para complicações não cardíacas em doentes com CIV isolada compa-

Tabela 2 - Distribuição das complicações cardíacas e não cardíacas nos doentes submetidos a cirurgia corretiva

\begin{tabular}{lc}
\hline Complicações cardíacas & $\mathbf{n}=\mathbf{8 5}(\%)$ \\
\hline Alterações de ritmo & $6(7,0)$ \\
Baixo débito & $6(7,0)$ \\
Derrame pericárdico & $3(3,5)$ \\
Paragem cardiorrespiratória & $2(2,4)$ \\
\hline Complicações não cardíacas & $\mathbf{n}=\mathbf{8 5}(\%)$ \\
\hline Pulmonares & $22(25,8)$ \\
Infeciosas & $15(17,7)$ \\
Ferida operatória & $6(7,1)$ \\
Neurológicas & $5(5,9)$ \\
Metabólicas & $4(4,7)$ \\
Hematológicas & $3(3,5)$ \\
Renais & $2(2,4)$ \\
Disfunção multiorgânica & $1(1,2)$ \\
\hline
\end{tabular}

rativamente às outras cardiopatias (teste da razão de verosimilhanças com $p=0,03$ ) e uma probabilidade 2,36 vezes maior para complicações não cardíacas em doentes com DCSAV comparativamente às outras cardiopatias (teste da razão de verosimilhanças $\operatorname{com} p=0,05)$.

A relação entre o diagnóstico de ToF e a ocorrência de complicações respiratórias foi estatisticamente significativa $\left(\chi^{2}, p=0,048\right)$, com um risco 6,6 vezes superior de ocorrência destas complicações (IC 95\%).

Apenas um doente na amostra teve necessidade de reoperação precoce. Seis doentes foram reoperados depois do primeiro mês após cirurgia corretiva: três doentes por CIV residual e três doentes por regurgitação da válvula aurículo-ventricular (AV) esquerda. O tempo decorrido entre a cirurgia corretiva e a reoperação tardia foi em média de 5,8 anos, com mediana de 3,2 anos (mínimo sete meses, máximo 16 anos). Um dos doentes submetido a valvuloplastia da válvula AV esquerda foi reoperado 16 anos mais tarde para colocação de prótese valvular mecânica.

Destes doentes, nenhum tinha sido submetido a cirurgia paliativa prévia à cirurgia corretiva.

Não houve correlação entre a ocorrência de HAP a médio prazo e a idade à data de cirurgia.

\section{Mortalidade}

A taxa de mortalidade a 30 dias do nosso estudo foi de $3 / 102$ casos $(2,9 \%)$. Dois casos faleceram no pós-operatório tardio, ambos no segundo mês após cirurgia (um dos casos após cirurgia corretiva e o segundo caso após cirurgia paliativa) (Fig. 3).

Quatro casos apresentavam DCSAV associado a outra cardiopatia [ToF $-n=1$, coartação da aorta (CoAo) $-n=1$, $\mathrm{CIA}-\mathrm{OS}-\mathrm{n}=2$ ] e o caso submetido apenas a cirurgia paliativa apresentava DCSAV. Não houve registo de mortalidade no grupo submetido a cateterismo terapêutico (Tabela 3).

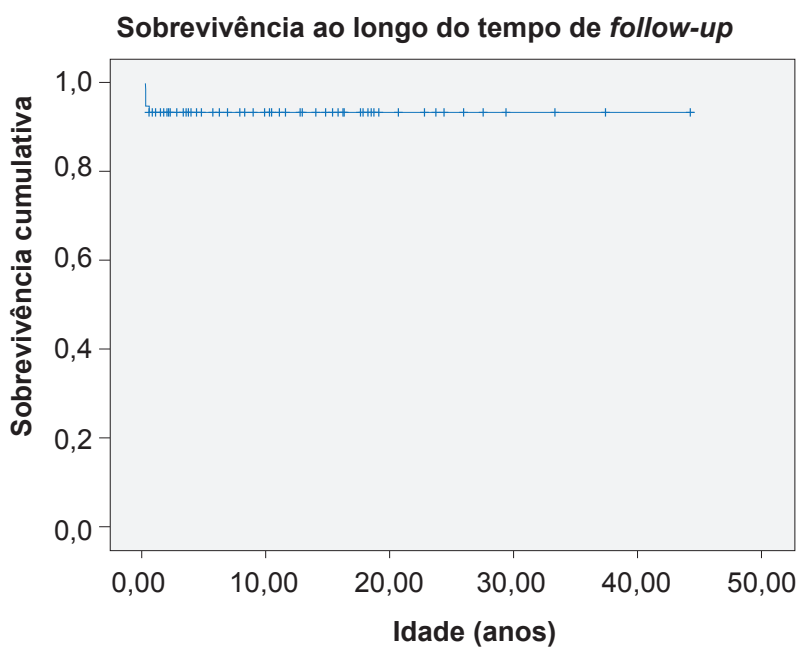

Figura 3 - Análise de Kaplan-Meier de sobrevida da amostra estudada. Todos os casos de mortalidade deste estudo ocorreram no primeiro ano de vida. Nos nossos doentes, ao longo do tempo total de follow-up, a idade média dos sobreviventes foi de 41,5 anos, com desvio-padrão de 1,2 anos (IC 95\%). 
Tabela 3 - Fatores de risco para mortalidade. Identificaram-se como fatores de risco para a ocorrência de mortalidade a presença global de complicações cardíacas e a ocorrência de baixo débito.

\begin{tabular}{lcc}
\hline Fatores de risco para mortalidade & Valor $\boldsymbol{p}$ & Odds ratio \\
\hline Complicações não cardíacas $^{\mathrm{a}}$ & 0,156 & \\
Complicações cardíacas $^{\mathrm{a}}$ & 0,016 & 17,3 \\
Baixo débito $^{\mathrm{a}}$ & 0,014 & 19,2 \\
Alterações de ritmo $^{\mathrm{a}}$ & 0,438 & \\
Ano de cirurgia $^{\mathrm{b}}$ & 0,131 & \\
Idade na cirurgia corretiva $^{\mathrm{b}}$ & 0,637 & \\
Peso na cirurgia corretiva $^{\mathrm{b}}$ & 0,547 & \\
\hline
\end{tabular}

a. Teste Qui-quadrado; b. Teste da razão de verosimilhanças.

Foi estudada a relação entre a ocorrência de complicações cardíacas e não cardíacas com a mortalidade a 30 dias. Verificou-se existência de relação estatisticamente significativa entre a mortalidade e a ocorrência de complicações cardíacas no pós-operatório imediato $\left(\chi^{2}\right.$, valor- $p=$ $0,005)$. Verificou-se uma relação significativa entre a ocorrência de baixo débito e a mortalidade a 30 dias $\left(\chi^{2}\right.$, valor $p=0,012$ ), com um risco de mortalidade (OR) de 39 vezes associado à ocorrência destas complicações (IC 95\%).

Não se demonstrou também relação estatística entre a ocorrência de complicações não cardíacas e a mortalidade a 30 dias.

A relação entre a mortalidade e o ano em que foi realizada a cirurgia não foi significativa ( $F$ da ANOVA, valor $p=$ $0,131)$. Não se verificou também correlação entre a idade ou o peso à data de cirurgia corretiva e a mortalidade.

\section{Seguimento pós-terapêutica}

Dos 102 doentes incluídos no estudo, 74 apresentavam registos de seguimento no nosso centro. As idades à data de registo da última consulta variaram entre quatro meses e 44 anos, sendo a mediana de 10 anos [IQR $(4,17)$ anos] (tempo de follow-up mínimo de um mês, máximo de 27,5 anos, mediana de seis anos). No total, seis doentes foram perdidos para follow-up.

\section{Sobrevida}

Cinquenta e cinco doentes operados tinham registos da sua classe funcional NYHA à data da última consulta. Destes, 49 doentes (89\%) estavam em classe funcional I, cinco doentes (9\%) em classe II e um doente (2\%) em classe III. No grupo que apenas realizou cateterismo terapêutico, todos os doentes se encontram em classe I da NYHA.

\section{DISCUSSÃO E CONCLUSÃO}

Este estudo, segundo o conhecimento dos autores, abrange o período de tempo mais longo de estudo de doentes com SD e cardiopatia congénita submetidos a terapêutica por cirurgia ou cateterismo relativamente a estudos anteriormente publicados.

A Associação Americana de Pediatria (AAP) publicou em 1994 as primeiras recomendações para o seguimento metódico e organizado de crianças com $\mathrm{SD},{ }^{9}$ nas quais se incluía a avaliação por um cardiologista pediátrico (mesmo na ausência de sopro) entre o nascimento e o final do primeiro mês de vida. Os nossos resultados demonstram que houve de facto uma alteração significativa da idade de referenciação após essa data, com uma idade cada vez mais precoce de encaminhamento, sobretudo para os doentes referenciados no primeiro ano de vida. Verificou-se que, na globalidade, mais de $50 \%$ dos doentes foram referenciados por sinais clínicos de doença cardíaca. Nos últimos anos, como seria de esperar, a referenciação por diagnóstico pré-natal de cardiopatia foi mais frequente.

É conhecida a preponderância dos defeitos septais nas cardiopatias congénitas das crianças com SD, 5,7,10-12 existindo uma grande variabilidade geográfica e étnica relativamente aos diagnósticos mais frequentes. Assim, enquanto em muitos estudos, sobretudo asiáticos, o defeito do septo interventricular é o diagnóstico mais frequente,,$^{10,13-14}$ os DSAV assumem em estudos europeus e norte-americanos o primeiro lugar. ${ }^{11}$ Estima-se que a maioria das crianças com DCSAV tenha trissomia $21 .{ }^{15} \mathrm{O}$ nosso estudo apresenta uma preponderância na prevalência dos DSAV (DCSAV $41 \%$, DISAV 4,9\%) relativamente aos outros diagnósticos, sendo a CIV o segundo diagnóstico mais frequente $(31,2 \%)$. Realça-se a associação de DCSAV e ToF, uma associação não muito frequente, que assume na nossa amostra uma prevalência de 3,9\%. Esta cardiopatia, de difícil abordagem cirúrgica, apresenta uma associação conhecida com o $\mathrm{SD}^{16}$ e provavelmente uma embriogénese particular nesta síndrome, uma vez que as duas cardiopatias apresentam origens embriogénicas muito distintas. ${ }^{16-17}$

Nove por cento dos doentes da nossa amostra (9/102) foram submetidos a cirurgia paliativa. Destes, quatro corresponderam a shunts sistémico-pulmonares no contexto de ToF e em cinco casos foi realizado banding da artéria pulmonar em doentes com DCSAV, por apresentarem ventrículos desproporcionados, muito baixo peso, outras patologias não cardíacas associadas, com aumento do risco cirúrgico, ou HAP grave. Nestes últimos, com resistências vasculares pulmonares no limite do risco cirúrgico aceitável (quatro a seis unidades Wood acima do primeiro ano de vida), procurou-se a diminuição do débito pulmonar para proteger a circulação pulmonar e avaliar a evolução das resistências com a redução do shunt, com vista a uma eventual correção cirúrgica posterior.

Os doentes submetidos apenas a cateterismo terapêutico apresentaram muito bons resultados em termos de classe funcional a longo prazo. Estes podem dever-se a uma boa seleção dos casos a submeter a cateterismo terapêutico, à menor complexidade da sua cardiopatia e à menor morbilidade associada a este procedimento, relativamente à abordagem cirúrgica.

É interessante verificar a necessidade cada vez menos frequente de realização de cateterismo cardíaco diagnóstico pré-cirúrgico a cada ano que passa, e presentemente a não realização de cateterismo no primeiro ano de vida para os defeitos septais e ToF. Este facto relaciona-se com 
a idade mais precoce de diagnóstico/referenciação e com o aperfeiçoamento das técnicas de ecocardiografia na definição da anatomia cardíaca e dos dados indiretos sobre a circulação pulmonar.

Tendo em conta que nos últimos anos se operaram vários adultos com SD e cardiopatia, não operados na infância, a tendência para uma idade mais precoce à data de cirurgia corretiva apenas foi verificada para os doentes com idade inferior a 12 meses. Esta tendência está de acordo com o descrito na literatura. , $11,18^{-18}$

Da mesma forma verificou-se que para os doentes com menos de $8 \mathrm{~kg}$, o peso foi cada vez menor à data da cirurgia, o que acompanha a tendência anterior. Não se encontrou, no entanto, relação estatística entre a idade ou o peso à data da cirurgia e a mortalidade.

Os doentes com SD apresentam no pós-operatório frequentemente maior morbilidade dos que doentes com as mesmas lesões mas sem SD, ${ }^{11,19}$ com maior taxa de complicações não cardíacas. De todas, as respiratórias estão descritas como sendo as mais frequentes, como o maior

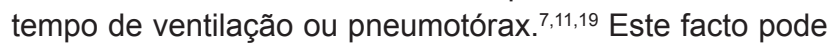
dever-se por um lado à maior taxa de obstrução crónica da via aérea, maior quantidade de secreções brônquicas e síndrome aspirativo crónico no contexto de refluxo gastroesofágico. ${ }^{7} \mathrm{O}$ nosso estudo corroborou esta maior prevalência de complicações respiratórias (25\%), seguidas das complicações infeciosas (18\%).

A ToF, apesar de ser uma das cardiopatias menos frequentes no SD, representando no nosso estudo $10,6 \%$ dos casos, associou-se à maior ocorrência de complicações respiratórias $(p=0,048)$, o que é difícil de explicar.

As complicações não cardíacas, apesar de frequentes (presentes em $51 \%$ dos doentes), foram na sua grande maioria alterações minor, e não se associaram a maior mortalidade. Esta constatação é sobreponível ao descrito noutros estudos. .,11,19 $^{-1}$

As complicações cardíacas foram mais raras do que as complicações não cardíacas (17,6\% dos doentes), não se verificando associação estatística entre si. Dentro das primeiras, as alterações de ritmo e o baixo débito cardíaco foram as mais frequentes, ocorrendo cada um destes tipos em $7 \%$ dos doentes. Desai et a ${ }^{\top}$ descrevem a taquicardia juncional ectópica como a disritmia mais frequente no pós-operatório da reparação de DCSAV. Embora menos frequentes, as complicações cardíacas apresentam relação estatisticamente significativa com a mortalidade no presente estudo.

No nosso estudo apenas um doente apresentou necessidade de reoperação precoce. Seis foram reoperados tardiamente, por CIV residual $(n=3)$ ou regurgitação significativa da válvula AV esquerda $(n=3)$.

O SD associa-se fortemente ao desenvolvimento de HAP e a síndrome de Eisenmenger, quer pela prevalência de cardiopatias com shunt esquerdo-direito, quer pela patologia respiratória obstrutiva. ${ }^{20}$ Quando comparados com doentes não SD com cardiopatia, os SD desenvolvem
HAP mais precocemente e têm pior capacidade funcional. ${ }^{20}$ O desenvolvimento de HAP irreversível pode ocorrer em doentes com DCSAV e SD sobretudo a partir dos seis a 12 meses de idade, pelo que presentemente se propõe a idade de seis meses, como limite ideal para abordagem cirúrgica corretiva desta patologia..$^{7,11,18}$

No nosso estudo, relativamente aos doentes submetidos a cirurgia cardíaca corretiva, a HAP pré-cirúrgica relacionou-se significativamente com a HAP precoce transitória $(p=0,03)$, mas não com HAP a médio prazo e persistente por doença vascular pulmonar. Assim, se a cirurgia for precoce, a HAP pré-cirúrgica grave pode prolongar-se no período pós-cirúrgico inicial, mas não representa obrigatoriamente alterações irreversíveis na vasculatura pulmonar.

A taxa de mortalidade a 30 dias no nosso estudo foi de $2,9 \%$, o que vai ao encontro dos valores encontrados noutros estudos. ${ }^{5,11} \mathrm{O}$ último óbito registado ocorreu em 1998 , pelo que a taxa de mortalidade a 30 dias entre 2000 e 2013 foi de $0 \%$. No entanto, a relação entre a mortalidade e o ano em que foi realizada a cirurgia não foi significativa. A análise da curva de sobrevivência de Kaplan-Meier (Fig. $3)$, permite-nos concluir que, após um período inicial pós-cirúrgico com maior mortalidade no primeiro ano de vida, os doentes com SD submetidos a cirurgia corretiva de cardiopatia congénita apresentam uma sobrevida a 45 anos sobreponível à da população geral.

Assim, a par de um aumento da prevalência de crianças nascidas com SD nos últimos 25 anos, verifica-se uma meIhoria dos cuidados de saúde prestados a estas crianças.

No nosso estudo, esta realidade espelha-se no maior número de casos com diagnóstico pré-natal, numa referenciação cada vez mais precoce para avaliação pelo cardiologista pediátrico e por fim, na qualidade dos resultados em termos de mortalidade e de capacidade funcional a longo prazo dos doentes submetidos quer a cirurgia corretiva, quer a cateterismo terapêutico.

\section{PROTECÇÃO DE PESSOAS E ANIMAIS}

Os autores declaram que os procedimentos seguidos estavam de acordo com os regulamentos estabelecidos pelos responsáveis da Comissão de Investigação Clínica e Ética e de acordo com a Declaração de Helsínquia da Associação Médica Mundial.

\section{CONFIDENCIALIDADE DOS DADOS}

Os autores declaram ter seguido os protocolos do seu centro de trabalho acerca da publicação de dados.

\section{CONFLITOS DE INTERESSE}

Os autores declaram não terem qualquer conflito de interesse relativamente ao presente artigo.

\section{FONTES DE FINANCIAMENTO}

Os autores declaram não ter recebido subsídios ou bolsas para a elaboração do artigo. 


\section{REFERÊNCIAS}

1. $\mathrm{OMIM}^{\circledR}$ - Online Mendelian Inheritance in $\mathrm{Man}^{\circledR}$ [homepage na Internet]. OMIM: \#190685. DOWN SYNDROME; [consultado 2015 out 02]. Disponível em: http://www.omim.org.

2. Loane M, Morris JK, Addor MC, Arriola L, Budd J, Doray B, et al. Twentyyear trends in the prevalence of Down syndrome and other trisomies in Europe: impact of maternal age and prenatal screening. Eur $\mathrm{J}$ Hum Genet. 2013;21: 27-33.

3. Ko JM. Genetic Syndromes associated with Congenital Heart Disease. Korean Circ J. 2015;45:357-61.

4. WebMD Health Professional Network - Medscape [homepage na Internet]. Harold Chen. Down Syndrome Clinical Presentation; [consultado 2015 out 02]. Disponível em: http://emedicine.medscape. com.

5. Evans JM, Dharmar M, Meierhenry E, Marcin JP, Raff GW. Association between Down syndrome and in-hospital death among children undergoing surgery for congenital heart disease. A US population-based study. Circ Cardiovasc Qual Outcomes. 2014;7:445-52

6. Champagne CR, Lewis M, Gilchrist DM. Should we mend their broken hearts? The history of cardiac repairs in children with Down syndrome. Pediatrics. 2014;134:1048.

7. Desai AR, Branco RG, Comitis GA, Maiya S, Vyas DB, Vaz Silva P. Early postoperative outcomes following surgical repair of complete atrioventricular septal defects: is Down syndrome a risk factor? Pediatr Crit Care Med. 2014;15:35-41.

8. St Louis JD, Jodhka U, Jacobs JP, He X, Hill KD, Pasquali SK, et al. Contemporary outcomes of complete atrioventricular septal defect repair: analysis of the Society of Thoracic Surgeons Congenital Heart Surgery Database. J Thorac Cardiovasc Surg. 2014;148:2526-31.

9. American Academy of Pediatrics Committee on Genetics. Health supervision for children with Down syndrome. Pediatrics. 1994;93:8559.

10. Freeman SB, Bean LH, Allen EG, Tinker SW, Locke AE, Druschel C. Ethnicity, sex, and the incidence of congenital heart defects: a report from the National Down Syndrome Project. Genet Med. 2008;10:173
80.

11. Tóth R, Szántó P, Prodán Z, Lex DJ, Sápi E, Szatmári A, et al. Down syndrome and postoperative complications after pediatric cardiac surgery: a propensity-matched analysis. Interact Cardiovasc Thorac Surg. 2013;17:691-7.

12. Elmagrpy Z, Rayani A, Shah A, Habas E, Aburawi EH. Down syndrome and congenital heart disease: why the regional difference as observed in the Libyan experience? Cardiovasc J Afr. 2011;22: 306-9.

13. Layangool T, Sangtawesin C, Kirawittaya T, Prompan W, Prachasilchai $P$, Pechdamrongsakul A. Survival analysis of Down syndrome with congenital heart disease: a 5-years registry at QSNICH. J Med Assoc Thai. 2014;97:S108-14.

14. Lal PS, Chavan B, Devendran VR, Varghese R, Murmu UC, Kumar RS. Surgical outcome of congenital heart disease in Down's syndrome. Asian Cardiovasc Thorac Ann. 2013;21:166-9.

15. Al-Hay AA, MacNeill SJ, Yacoub M, Shore DF, Shinebourne EA. Complete atrioventricular septal defect, Down syndrome, and surgical outcome: risk factors. Ann Thorac Surg. 2003;75:412-21.

16. Vergara P, Digilio MC, De Zorzi A, Di Carlo D, Capolino R, Rimini A, et al. Genetic heterogeneity and phenotypic anomalies in children with atrioventricular canal defect and tetralogy of Fallot. Clin Dysmorphol. 2006;15:65-70.

17. Nguyen $\mathrm{HH}$, Jay PY. A single misstep in cardiac development explains the co- occurrence of tetralogy of Fallot and complete atrioventricular septal defect in Down syndrome. J Pediatr. 2014;165:194-6. doi:

18. Kobayashi M, Takahashi $\mathrm{Y}$, Ando M. Ideal timing of surgical repair of isolated complete atrioventricular septal defect. Interact Cardiovasc Thorac Surg. 2007;6:24-6.

19. Tumanyan MR, Filaretova OV, Chechneva VV, Gulasaryan RS, Butrim IV, Bockeria LA. Repair of complete atrioventricular septal defect in infants with down syndrome: outcomes and long-term results. Pediatr Cardiol. 2015;36:71-5.

20. D'Alto M, Mahadevan VS. Pulmonary arterial hypertension associated with congenital heart disease. Eur Respir Rev. 2012;21:328-37. 